\title{
Oceanography of the Indonesian Seas
}

THE GLOBAL OCEAN is composed of parts—its regions-each playing a role in the global scheme of the ocean and climate systems. Each region has its intrinsic interest and uniqueness. Of course, the same physics applies to all, but by virtue of position, topographic constraints, and coupling to the atmosphere, some regions attract more interest than others; some are more central to the larger-scale systems. The tropical seas of Indonesia are such a place. There, Pacific water flows into the Indian Ocean amid a complex array of islands and of deep and shallow seas connected by narrow passages of varied depths. Stratification is subject to intense tidal-induced mixing, all under an atmosphere regulated by the Asian-Australian monsoons and subject to the whims of the El Niño Southern Oscillation. Understanding the interplay of these variables provides a challenge that oceanographers can't resist.

I often wonder what oceanographers of the first half of the $20^{\text {th }}$ century would think of their quest to map the ocean if they could see the data that we now have. Their maps of ocean properties were drawn from hydrographic stations $100 \mathrm{~km}$ apart, with samples at depth intervals of tens or hundreds of meters, with little regard for the time of year. Each data point was obtained with enormous effort. No doubt that the value placed on each precious data point made them look harder at their scant data. They had no choice but to assume steady state, an assumption they knew was weak, but it was a practical assumption that did allow for some exciting discoveries. But if they would see the swirls and intricate texture of the ocean properties as revealed by a few good images from space of sea surface temperature, wind, chlorophyll, or sea-level heights, or if they could see the temporal variability exposed by arrays of moorings packed with sensors, would they have just given up making their maps? Of course not. It's not in the nature of explorers to wait for better apparatus the future might eventually provide. One goes forth with the tools at hand.

This special issue of Oceanography attempts to convey the uniqueness of the Indonesian seas and of the progress that has been made in recent years in understanding their oceanography, with what will eventually be viewed as the crude tools of the opening years of the $21^{\text {st }}$ century.

The advances brought about by remote sensing from space is a central theme of many of the contributions, but to better appreciate these new views, a bit of the history of ocean exploration of the Indonesian seas is presented. Progress is built upon the hard work and ideas of our predecessors.

I appreciate the timely response of the authors, the editing skills of Ellen Kappel, and the support provided by the U.S. National Aeronautics and Space Administration (NASA) to produce this issue.

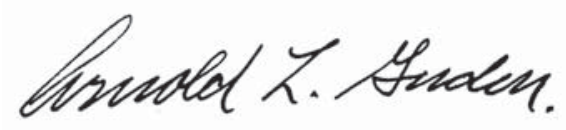

ARNOLD L. GORDON 